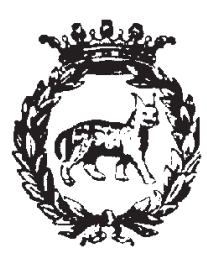

Partial Differential Equations - Regularity of optimal transport maps and partial differential inclusions, by Luigi Ambrosio, Guido De Philippis and Bernd KirchHeIM, communicated on 13 May 2011.

\title{
Dedicated to Giovanni Prodi.
}

\begin{abstract}
In this work we address the issue of Sobolev regularity of solutions of MongeAmpere equations in a borderline case and show how this question is related to a rigidity problem for partial differential inclusions.
\end{abstract}

KeY WORds: Monge-Ampere equations, optimal transportation, partial differential inclusions.

Mathematics Subject Classification: 35D10, 49Q20.

\section{INTRODUCTION}

In this paper we deal with the regularity of planar optimal transport maps, in a "critical" case not covered presently in the literature. Following a suggestion by J. Maly, and the ideas in [1], we relate the regularity problem to a "rigidity" problem for partial differential inclusions which might be interesting in its own right.

Let us start with the first problem. We give a formulation in terms of subdifferentials (i.e. cyclically monotone operators), deferring the relations with optimal transport theory to Section 6 .

Problem 1. Let $u: \mathbb{R}^{2} \rightarrow P\left(\mathbb{R}^{2}\right)$ be a subdifferential, $A \subset \operatorname{Dom}(u)$ open, and let us assume that

$$
\frac{1}{L} \mathscr{L}^{2}\left\llcorner A \leq J u\left\llcorner A \leq L \mathscr{L}^{2}\llcorner A .\right.\right.
$$

for some $L>1$. Show that $u$ is a locally Sobolev map.

The Jacobian measure $J u$ appearing in the statement of Problem 1 is the Monge-Ampére measure of the potential function $\varphi$ whose subdifferential coincides with $u$, i.e.

$$
u(x)=\partial \varphi(x)=\left\{p \in \mathbb{R}^{2}: \varphi(y) \geq \varphi(x)+\langle p, y-x\rangle \forall y\right\} .
$$


In order to state problem 2, we need to define the set of "admissible" gradients

$$
\mathcal{A}:=\left\{M \in \operatorname{Sym}^{2 \times 2}:\|M\| \leq 1,(L+1)|\operatorname{Trace}(M)| \leq(L-1)(1+\operatorname{det}(M))\right\},
$$

where $\|\cdot\|$ is the operator norm, and the subset $\mathcal{S}$ of "singular" gradients is defined by

$$
\mathcal{S}:=\left\{R^{-1}\left(\begin{array}{cc}
1 & 0 \\
0 & -1
\end{array}\right) R: R \in S O(2)\right\} .
$$

Problem 2. Let $B \subset \mathbb{R}^{2}$ be a connected open set, $f: B \rightarrow \mathbb{R}^{2}$ Lipschitz, and assume that $D f \in \mathcal{A} \mathscr{L}^{2}$-a.e. in B. Show that if the set

$$
\{x \in B: D f(x) \in \mathcal{S}\}
$$

has positive $\mathscr{L}^{2}$-measure, then $f$ is locally affine.

Some comments on Problems 1 and 2 are in order. As we will see in Section 6, maps $u=\partial \varphi$ as in Problem 1 appear in a natural way in the optimal transport problem, in the case when the target measure has a convex support. In this situation Caffarelli made clear in [4] the connection with Alexandrov (and viscosity) solutions to Monge-Ampére equations Det $\nabla^{2} \varphi=f$. His regularity theory, developed in a series of papers $[4,5,6]$, and in any number of dimensions, provides Hölder continuity of $u$; since we use continuity in some proofs, to make our paper self-contained we provide a brief proof of continuity, based on a simple duality argument, in Section 5. On the other hand, since $u$ is the gradient of a convex function, it has $B V$ regularity. So, Problem 1 is equivalent to ruling out a "Cantor" part of the distributional derivative, a question for which no standard procedure is available. This fine regularity question arises, for instance, in evolution problems where the instantaneous coupling between velocity and density is given by an optimal transport map (see [3], [7]); in this case a positive answer to Problem 1 would give an optimal regularity result for the velocity.

The differential inclusion considered in problem 2 is an interesting variant of the typical one, where one requires a Lipschitz map $f$ to take gradients in a prescribed compact set $K$ and one possibly imposes affine Dirichlet boundary conditions $f(x)=A x$ on the boundary of the domain (see for instance [13] [14], for a nice presentation of the theory); in this situation one calls the set $K$ rigid if the only solutions are affine. Also, it is well-known that a necessary, but not sufficient, rigidity condition is the absence of rank-one connections inside $K$. In our case we are considering the partial differential inclusion with $K=\mathcal{A}$ but we are at the same time requiring that the smaller set $\mathcal{S}$ of gradients is attained in a set of positive measure. Since it is not difficult to show (see Lemma 7.1) that no rankone connection exists between matrices in $\mathcal{S}$ and matrices in $\mathcal{A}$ we can hope for a rigidity result.

The equivalence between Problems 1 and 2 is provided by Minty's correspondence between graphs of 1-Lipschitz maps and graphs of monotone operators. This correspondence, induced by the " $\pi / 4$ rotation in $\mathbb{R}^{2}$ " defined in $(3)$, is heavily exploited in [1] to derive several fine properties of monotone operators. 
At this stage both problems, as stated, are open. We hope that the connection between them will lead to some progress, either with a positive result or with a counterexample. We also hope, in future work, to study the relation between these problems in higher dimensions. The plan of the paper is the following. In Section 1 we recall the main measure-theoretic notation and the basic facts on currents associated to Lipschitz graphs. In Section 2 we state the main properties of monotone maps $u$, introducing the Jacobian measure $J u$, the Cayley transformation and the canonical current $G_{u}$ associated to $u$, along the lines of [1]. In Section 3 and 4 we relate the singular part of the distributional derivative $D u$ to the set of "singular" gradients $\mathcal{S}$ in (2) and, at the same time, we relate bounds on the Jacobian measure $J u$ to the set of admissible gradients $\mathcal{A}$ in (1). Using these informations, in Section 6 we prove the equivalence of the two problems and relate Problem 1 to optimal transport theory, providing also some additional motivation for it. Finally in Section 7 we show some properties of a potential solution of Problem 2.

Acknowledgement. We thank A. Figalli and J. Maly for very useful discussions on the questions discussed in this paper. Work supported by the ERC ADG Grant GeMeThNES.

\section{Notation AND PRELIMINARy RESUlts}

In this paper we denote by $\left(e_{1}, e_{2}\right)$ the canonical basis of $\mathbb{R}^{2}$; since we will often consider maps from $\mathbb{R}^{2}$ to $\mathbb{R}^{2}$, we prefer to use a distinguished notation $\left(\varepsilon_{1}, \varepsilon_{2}\right)$ for the canonical basis in the target space $\mathbb{R}^{2}$. Also, when using the Cayley transformation

$$
\left\{\begin{array}{l}
x^{\prime}=\frac{x+y}{\sqrt{2}} \\
y^{\prime}=\frac{-x+y}{\sqrt{2}}
\end{array}\right.
$$

we shall always use a ' to denote the canonical bases in the new system of coordinates. Hence, $\left(e_{1}^{\prime}, e_{2}^{\prime}\right)$ and $\left(\varepsilon_{1}^{\prime}, \varepsilon_{2}^{\prime}\right)$ are respectively the canonical bases in

$$
\Delta:=\left\{(x, y) \in \mathbb{R}^{2} \times \mathbb{R}^{2}: x=y\right\}, \quad \Delta^{\perp}:=\left\{(x, y) \in \mathbb{R}^{2} \times \mathbb{R}^{2}: x=-y\right\} .
$$

These bases are related by

$$
\left\{\begin{array}{l}
e_{1}^{\prime}=\frac{1}{\sqrt{2}}\left(e_{1}+\varepsilon_{1}\right) \\
e_{2}^{\prime}=\frac{1}{\sqrt{2}}\left(e_{2}+\varepsilon_{2}\right) \\
\varepsilon_{1}^{\prime}=\frac{1}{\sqrt{2}}\left(-e_{1}+\varepsilon_{1}\right) \\
\varepsilon_{2}^{\prime}=\frac{1}{\sqrt{2}}\left(-e_{2}+\varepsilon_{2}\right) .
\end{array}\right.
$$

The corresponding dual bases shall be denoted by $d x^{1}, d x^{2}, d y^{1}, d y^{2}$ and, in the rotated system of coordinates, by $d x^{\prime 1}, d x^{\prime 2}, d y^{\prime 1}, d y^{\prime 2}$. 
We denote with $\mathscr{L}^{n}$ the Lebesgue measure on $\mathbb{R}^{n}$ and with $\mathscr{H}^{k}$ the $k$-dimensional Hausdorff measure. Recall that for any $k$-dimensional subspace $V \subset \mathbb{R}^{n}, \mathscr{H}^{k}\llcorner V$ coincides with the Lebesgue measure on $V$, where for any Radon measure $\mu$ and any Borel set $B, \mu\llcorner B(A)$ is given by $\mu(A \cap B)$. If $f: X \rightarrow Y$ is a Borel map we denote by $f_{\#} \mu$ the push-forward of $\mu$, i.e. the measure $f_{\#} \mu(B)=\mu\left(f^{-1}(B)\right)$. We shall occasionally use the fact that continuous images of Borel sets, though not Borel in general, are measurable with respect to any positive finite Borel measure; this fact allows to avoid the use of tedious inner approximations by compact sets.

If $A \subset \mathbb{R}^{n}$ is open and $u \in B V_{\text {loc }}\left(A ; \mathbb{R}^{m}\right)$ is a map of bounded variation, we denote with $D u$ its distributional derivative (a $m \times n$-matrix valued measure) and with $\nabla u$ its absolutely continuous part with respect to the Lebesgue measure. We recall also that any $B V$ map is approximately differentiable $\mathscr{L}^{n}$-almost everywhere in its domain and that its approximate differential coincides with $\mathscr{L}^{n}$-a.e. with $\nabla u$ (see [2]). If $u \in W^{1, p}$ with $p \in[1, \infty]$ we are going to use, with a slight abuse of notation, the symbol $D u$ to denote both the distributional derivative (i.e., strictly speaking a measure) and the weak derivative.

\subsection{Currents and graphs of Lipschitz maps}

Recall that a 2-current $T$ in $\mathbb{R}^{4}, T \in \mathcal{D}_{2}\left(\mathbb{R}^{4}\right)$, is a linear continuous functional on the space $\mathcal{D}^{2}\left(\mathbb{R}^{4}\right)$ of compactly supported 2-forms in $\mathbb{R}^{4}$. We will denote the action of $T$ on a smooth form $\omega$ both with $\langle T, \omega\rangle$ and with $T(\omega)$. We write $T_{k} \stackrel{*}{\rightarrow} T$ if

$$
T_{k}(\omega) \rightarrow T(\omega) \text { for any 2-form } \omega
$$

A current $T$ is said to be rectifiable, $T \in \mathcal{R}_{2}\left(\mathbb{R}^{4}\right)$, if its action on 2-forms $\omega$ can be expressed as:

$$
T(\omega)=\int_{M}\left\langle\omega(x), \xi_{M}(x)\right\rangle \theta(x) d \mathscr{H}^{k}(x),
$$

where $M \subset \mathbb{R}^{4}$ is a countably $\mathscr{H}^{2}$-rectifiable set, $\xi_{M}(x)$ is a simple 2-vector orienting the approximate tangent space ap $-T_{x} M$ and $\theta(x)$ is an integer-valued positive function, see [8] for precise definitions.

If $f: \mathbb{R}^{2} \rightarrow \mathbb{R}^{2}$ is a Lipschitz map, we shall denote by

$$
|\mathcal{M}(D f)|=\sqrt{1+|D f|^{2}+\operatorname{det}^{2}(D f)}
$$

the area element of $f$. Thanks to Federer's area formula [8, Theorem 3.2.3], it can be used to compute the area of the graph $\Gamma_{f}$ of $f$. More precisely, for every Borel set $B \subset \mathbb{R}^{2}$ it holds

$$
\mathscr{H}^{2}(\tilde{B})=\int_{B}|\mathcal{M}(D f)| d x
$$


where $\tilde{B}=\{(x, y): y=f(x)\}$ is corresponding set on $\Gamma_{f}$. Denoting also

$$
\mathcal{M}(D f):=\left(e_{1}+d f\left(e_{1}\right)\right) \wedge\left(e_{2}+d f\left(e_{2}\right)\right)
$$

the definition in (6) is consistent with (8), namely the area factor is precisely the modulus of the simple 2-vector $\mathcal{M}(D f)$.

If $f=f^{1} \varepsilon_{1}+f^{2} \varepsilon_{2}$, we have

$$
\begin{aligned}
\mathcal{M}(D f)= & \left(e_{1}+D_{e_{1}} f^{1} \varepsilon_{1}+D_{e_{1}} f^{2} \varepsilon_{2}\right) \wedge\left(e_{2}+D_{e_{2}} f^{1} \varepsilon_{1}+D_{e_{2}} f^{2} \varepsilon_{2}\right) \\
= & e_{1} \wedge e_{2}+D_{e_{2}} f^{1} e_{1} \wedge \varepsilon_{1}+D_{e_{2}} f^{2} e_{1} \wedge \varepsilon_{2} \\
& -D_{e_{1}} f^{1} e_{2} \wedge \varepsilon_{1}-D_{e_{1}} f^{2} e_{2} \wedge \varepsilon_{2}+\operatorname{det} D f \varepsilon_{1} \wedge \varepsilon_{2} .
\end{aligned}
$$

We shall also denote by $G_{f}$ the canonical 2-current associated to $\Gamma_{f}$. It is the push-forward of the standard 2-current $\left[\mathbb{R}^{2}\right]$ in $\mathbb{R}^{2}$ under the map $x \mapsto$ $(I d \times f)(x)$, namely

$$
\left\langle G_{f}, \omega\right\rangle=\left\langle\left[\mathbb{R}^{2}\right],(I d \times f)^{\#} \omega\right\rangle
$$

for any smooth 2 -form $\omega$. It is well known [10, Section 3.2] that $G_{f}$ can be represented as

$$
\left\langle G_{f}, \omega\right\rangle=\int_{\Gamma_{f}}\left\langle\xi_{f}(x, y), \omega(x, y)\right\rangle d \mathscr{H}^{2}(x, y)
$$

(the bracket inside the integral being the duality between vectors and covectors) where $\xi_{f}$, the so-called orienting vectorfield of the current, is a unit simple 2-vector linked to $f$ by the relation

$$
\xi_{f}(x, f(x))=\frac{\mathcal{M}(D f(x))}{|\mathcal{M}(D f(x))|} .
$$

Since $\Gamma_{f}$ is better than a countably $\mathscr{H}^{2}$-rectifiable set, namely a Lipschitz graph, an immediate consequence of the Rademacher theorem is that for $\mathscr{H}^{2}\left\llcorner\Gamma_{f}\right.$ almost every $\left(x_{0}, y_{0}\right)$ there exists a classical tangent space to $\Gamma_{f}$, and coincides with the approximate tangent space. This means that there exists a 2-plane $P$ such that, if we denote with $\pi_{P}$ the orthogonal projection on $P$,

$$
\lim _{(x, y) \rightarrow\left(x_{0}, y_{0}\right),(x, y) \in \Gamma_{f}} \frac{\left|\left(x-x_{0}, y-x_{0}\right)-\pi_{P}\left(x-x_{0}, y-x_{0}\right)\right|}{\sqrt{\left|x-x_{0}\right|^{2}+\left|y-y_{0}\right|^{2}}}=0 .
$$

We will denote this plane as $T_{\left(x_{0}, y_{0}\right)} \Gamma_{f}$, oriented by the unit simple 2-vector $\xi_{f}$ in (10).

To write our formulas in components, let

$$
\omega=\omega^{20} d x^{1} \wedge d x^{2}+\sum_{i, j=1}^{2} \omega^{\overline{i j}} d x^{\bar{i}} \wedge d y^{j}+\omega^{02} d y^{1} \wedge d y^{2},
$$


where $\bar{i}$ is the complement of $i$ in $\{1,2\}$. Then, the action of $G_{f}$ on $\omega$ is

$$
\begin{aligned}
G_{f}(\omega)= & \int_{\mathbb{R}^{2}} \omega^{20}(x, f(x))+\sum_{i, j=1}^{2}(-1)^{i} \omega^{\overline{i j}}(x, f(x)) D_{i} f^{j}(x) \\
& +\omega^{02}(x, f(x)) \operatorname{det} D f(x) d x
\end{aligned}
$$

Currents with locally finite mass can be canonically extended to bounded Borel forms with compact support; this way, (11) remains true in this more general class of forms. Moreover, since $f$ is locally bounded the mass is finite in products $K \times \mathbb{R}^{2}$ with $K$ compact; hence the forms can be taken to be supported in $K \times \mathbb{R}^{2}$ with $K$ compact subset of $\mathbb{R}^{2}$. For any Borel set $B \subset \mathbb{R}^{4}$ we can also define

$$
G_{f}\left\llcorner B(\omega)=\int_{B \cap \Gamma_{f}}\left\langle\xi_{f}, \omega\right\rangle d \mathscr{H}^{2} .\right.
$$

\section{Monotone maps And CAyley transformation}

We recall that a multivalued map $u: \mathbb{R}^{2} \rightarrow P\left(\mathbb{R}^{2}\right)$ is said to be monotone if

$$
\langle p-q, x-y\rangle \geq 0 \quad \forall p \in u(x), q \in u(y) .
$$

Notice that $u$ is monotone if and only if its inverse $u^{-1}(p)=\{x: p \in u(x)\}$ is monotone. Among monotone maps, a distinguished role is played by subdifferentials $\partial \varphi$ of convex, proper and lower semicontinuous functions $\varphi: \mathbb{R}^{2} \rightarrow \mathbb{R} \cup$ $\{+\infty\}$, namely

$$
\partial \varphi(x):=\left\{p \in \mathbb{R}^{2}: \varphi(y) \geq \varphi(x)+\langle p, y-x\rangle \forall y\right\}
$$

if $\varphi(x)<+\infty$, and $\partial \varphi(x)=\emptyset$ otherwise.

In the case when $u=\partial \varphi$ it is easily seen that $u^{-1}=\partial \varphi^{*}$, where $\varphi^{*}$ is the conjugate function of $\varphi$ :

$$
\varphi^{*}(y):=\sup _{x \in \mathbb{R}^{2}}\langle x y\rangle-\varphi(x)
$$

We shall denote by $\operatorname{Dom}(u)$ the domain of $u$, i.e. the set of points $x$ such that $u(x) \neq \emptyset$, and by $\Gamma_{u} \subset \mathbb{R}^{2} \times \mathbb{R}^{2}$ its graph, i.e. the set $\{(x, y): y \in u(x)\}$. We recall (see [1] for a simple proof) that the set of points $x$ such that $u(x)$ contains more than one point is $\sigma$-finite w.r.t. $\mathscr{H}^{1}$; in particular it is $\mathscr{L}^{2}$-negligible. Moreover, the complement of this set, that we shall denote by $\mathcal{C}_{u}$, is made by continuity points of $u$, namely

$$
x_{k} \rightarrow x, x_{k} \in \operatorname{Dom}(u), p_{k} \in u\left(x_{k}\right) \text { implies } p_{k} \rightarrow u(x) \quad \forall x \in \mathcal{C}_{u}
$$


The Jacobian measure $J u$ of a monotone map $u$ is defined on Borel sets of $\mathbb{R}^{2}$ by

$$
J u(B)=\mathscr{L}^{2}\left(\bigcup_{x \in B} u(x)\right)
$$

It can be easily shown that $J u$ is $\sigma$-additive, because the images of disjoint sets have a $\mathscr{L}^{2}$-negligible intersection (indeed, this intersection is contained in the set of points where $u^{-1}$ is multi-valued). In addition it is apparent from the definition that $J u$ is local, namely $J u\llcorner B=J v\llcorner B$ whenever $B$ is Borel and $u=v$ in $B$. In the case when $u=\partial \varphi$ the measure $J u$, formally correspondent to $\operatorname{det} \nabla^{2} \varphi$, is called Monge-Ampére measure of $\varphi$.

Minty noticed that we can put in 1-1 correspondence monotone functions $u$ and 1-Lipschitz functions $f$ via the Cayley transformation $\Phi: \mathbb{R}^{2} \rightarrow \mathbb{R}^{2}$ defined in (3), so that

$$
\frac{p-x}{\sqrt{2}}=f\left(\frac{p+x}{\sqrt{2}}\right) \quad \forall p \in u(x) .
$$

At the level of maximal monotone maps $u: \mathbb{R}^{2} \rightarrow P\left(\mathbb{R}^{2}\right)$ (where maximality is defined with respect to inclusion of graphs) we have 1-1 correspondence with graphs of entire 1-Lipschitz maps $f: \Delta \rightarrow \Delta^{\perp}$, via the relation $\Gamma_{f}=\Phi\left(\Gamma_{u}\right)$.

With a slight abuse of notation, at all points $x$ where $u$ is single-valued we denote by $u(x)$ the unique element of the set, so that the functions $f$ and $u$ are related by

$$
\frac{u(x)-x}{\sqrt{2}}=f\left(\frac{u(x)+x}{\sqrt{2}}\right) .
$$

at all points where $u$ is single-valued.

In [1] the geometric and analytic implications of this have been widely studied. First of all, notice that since the graph $\Gamma_{f}$ of a Lipschitz map has a natural structure of multiplicity one current without boundary and $\Gamma_{f}=\Phi\left(\Gamma_{u}\right)$, we can define the current

$$
\left\langle G_{u}, \omega\right\rangle:=\left\langle G_{f},\left(\Phi^{-1}\right)^{\#}(\omega\rangle\right) .
$$

Heuristically, $G_{u}$ is associated to the integration on the graph of $u$, including vertical parts. As for $J u$, the action of $G_{u}$ is local, i.e. $\left\langle G_{u}, \omega\right\rangle=\left\langle G_{v}, \omega\right\rangle$ whenever $u, v$ are monotone, $u \equiv v$ in an open set $A$ and $\omega$ is supported in $A \times \mathbb{R}^{2}$.

The orientation $\xi_{u}$ of the current $G_{u}$ is induced in the natural way from the orientation $\xi_{f}$ in $(10)$ of $G_{f}$, via $\Phi$. The following definition will play an important role in our discussion.

Definition 3.1 (Vertical and totally vertical points). Let us call $V$ the vertical points of the graph of $u$, i.e

$$
V=\left\{(x, y) \in \Gamma_{u}: \xi_{u}^{20}(x, y)=0\right\}
$$


We say that a point $(x, y) \in V$ is totally vertical if also $\xi_{u}^{02}(x, y)$ vanishes and denote by $V_{u}$ the collection of these points.

Thanks to the area formula for maps defined on rectifiable sets (see [8, Corollary 3.2.20]), it is easy to see that $\xi_{u}^{20}$ equals the area factor of the projection $\pi_{1}: \Gamma_{u} \rightarrow \mathbb{R}^{2} \times\{0\}$, i.e.

$$
\mathscr{H}^{2}\left(\pi_{1}(B)\right)=\int_{B}\left|\xi_{u}^{20}\right| d \mathscr{H}^{2} \quad \text { for all Borel sets } B \subset \Gamma_{u},
$$

while $\xi^{02}$ equals the area factor of the projection $\pi_{2}: \Gamma_{u} \rightarrow\{0\} \times \mathbb{R}^{2}$ :

$$
\mathscr{H}^{2}\left(\pi_{2}(B)\right)=\int_{B}\left|\xi_{u}^{02}\right| d \mathscr{H}^{2} \quad \text { for all Borel sets } B \subset \Gamma_{u} .
$$

The main properties of $G_{u}$ proved in [1] are summarized in the following two theorems.

THEOREM 3.2. The following properties hold:

(i) If $\left(u_{k}\right)$ and $u$ are maximal monotone and $\Gamma_{u_{k}} \rightarrow \Gamma_{u}$ in the Kuratowski sense, then $G_{u_{k}} \stackrel{*}{\rightarrow} G_{u}$ as currents. If $u$ is single-valued (and therefore continuous) in an open set $A$, then convergence of $u_{k}$ to $u$ is locally uniform in $A$.

(ii) The maps $u_{\varepsilon}:=\left(\varepsilon I+u^{-1}\right)^{-1}$ are single-valued, Lipschitz, maximal monotone with $\operatorname{Dom}\left(u_{\varepsilon}\right)=\mathbb{R}^{n}$ and converge to $u \mathscr{L}^{2}$-a.e. in $\operatorname{Dom}(u)$. Moreover, if $u=\partial \varphi$, then $u_{\varepsilon}=D \varphi_{\varepsilon}$, where $\varphi_{\varepsilon}(x):=\inf _{y}\left\{\varphi(y)+|x-y|^{2} /(2 \varepsilon)\right\}$ is the infconvolution approximation of $\varphi$.

TheOREM 3.3. Let $u: \mathbb{R}^{2} \rightarrow P\left(\mathbb{R}^{2}\right)$ be a maximal monotone map and let $\Omega$ be the interior of its domain. Then the following assertions hold:

(i) $u \in B V_{\text {loc }}\left(\Omega ; \mathbb{R}^{2}\right), \Gamma_{u}$ has locally finite $\mathscr{H}^{2}$-measure in $\Omega \times \mathbb{R}^{2}$ and

$$
|D u|(B) \leq \mathscr{H}^{2}\left(\Gamma_{u} \cap\left(B \times \mathbb{R}^{2}\right)\right)
$$

for every Borel set $B \subset \Omega$.

(ii) Let

$$
\mathcal{D}=\left\{(x, y) \in \Gamma_{u} \text { such that } T_{(x, y)} \Gamma_{u} \text { exists }\right\}
$$

Then for every $x \in \Omega \cap \pi_{1}(\mathcal{D} \backslash V)$ the map $u$ is differentiable, with gradient $\nabla u(x)$, in the following sense:

$$
\lim _{y \rightarrow x, p \in u(y)} \frac{|p-u(x)-\langle\nabla u(x), y-x\rangle|}{|y-x|}=0 .
$$

In addition, $\pi_{1}(\mathcal{D} \backslash V)$ has full Lebesgue measure in $\Omega, \nabla u$ is the density of $D u$ with respect to $\mathscr{L}^{2}$ and is symmetric if $u$ is a subdifferential. 
(iii) The density of $J u\left\llcorner\Omega\right.$ with respect to $\mathscr{L}^{2}$ coincides with $\operatorname{det} \nabla u \mathscr{L}^{2}$-a.e. in $\Omega$.

(iv) If $u_{\varepsilon}$ are defined as in Theorem 3.2(ii), then $\left|D u_{\varepsilon}\right| \stackrel{*}{\rightarrow}|D u|$ and $J u_{\varepsilon}=\operatorname{det} D u_{\varepsilon} \rightarrow$ Ju as measures in $\Omega$.

A simple consequence of the previous properties is the following relation between $G_{u}$ and $J u$, see also [1, Theorem 5.11]:

$$
\langle J u, \varphi\rangle=\left\langle G_{u}, \varphi(x) d y^{1} \wedge d y^{2}\right\rangle
$$

for any bounded Borel function with compact support. Coming back to Minty's transformation, the following proposition will also be useful.

Proposition 3.4. Let $u$ be a maximal monotone function, then

(i) If $u=\partial \varphi$ on $\operatorname{Dom}(u)$ for some convex function, then $D f$ is symmetric.

(ii) Conversely if $D f$ is symmetric and $\operatorname{Int}(\operatorname{Dom}(u)) \neq \emptyset$ then $u=\partial \varphi$ for some convex function $\varphi$ on $\operatorname{Int}(\operatorname{Dom}(u))$.

ProOf. By (13), the first implication is trivial if $\varphi \in C^{1,1}\left(\mathbb{R}^{n}\right)$. Consider now the approximations provided by the point (iii) of Theorem 3.2, in correspondence to this approximation there are Lipschitz gradients $f_{\varepsilon}$ such that $\Phi\left(\Gamma_{f_{\varepsilon}}\right)=\Gamma_{u_{\varepsilon}}$. Since $G_{u_{\varepsilon}}$ weakly converge to $G_{u}$, it follows that $G_{f_{\varepsilon}}$ weakly converge to $\left(\Phi^{-1}\right)^{\#}\left(G_{u}\right)$. This convergence of graphs easily implies, by compactness, that $f_{\varepsilon}$ weak-* converge in $W^{1, \infty}$ to the function $f$ such that $\Gamma_{u}=\Phi\left(\Gamma_{f}\right)$. Since weak-* convergence preserves the symmetry of distributional derivatives, we obtain the claim.

For notational simplicity (and since we are going to use it only in this case) we prove the second implication only for $n=2$. Thanks to [1, Equation (5.5)] for any $\varphi \in C_{c}^{\infty}\left(\mathbb{R}^{2}\right)$ we have

$$
\int D_{1} \varphi(x) u^{2}(x) d x=-\left\langle G_{u}, \varphi(x) d x^{2} \wedge d y^{2}\right\rangle
$$

and

$$
\int D_{2} \varphi(x) u^{1}(x) d x=\left\langle G_{u}, \varphi(x) d x^{1} \wedge d y^{1}\right\rangle .
$$

Thanks to equations (11), (14) and (27) we have

$$
\begin{aligned}
-\left\langle G_{u}, \varphi(x) d x^{2} \wedge d y^{2}\right\rangle & =-\left\langle G_{f}, \varphi\left(\frac{x^{\prime}+y^{\prime}}{\sqrt{2}}\right) d x^{\prime 2} \wedge d y^{\prime 2}\right\rangle \\
& =\int \varphi\left(x^{\prime}+f\left(x^{\prime}\right)\right) D_{1} f^{2}\left(x^{\prime}\right) d x^{\prime}
\end{aligned}
$$


and

$$
\begin{aligned}
\left\langle G_{u}, \varphi(x) d x^{1} \wedge d y^{1}\right\rangle & =\left\langle G_{f}, \varphi\left(\frac{x^{\prime}+y^{\prime}}{\sqrt{2}}\right) d x^{\prime 1} \wedge d y^{\prime 1}\right\rangle \\
& =\int \varphi\left(x^{\prime}+f\left(x^{\prime}\right)\right) D_{2} f^{1}\left(x^{\prime}\right) d x^{\prime}
\end{aligned}
$$

Since $D_{2} f^{1}=D_{1} f^{2}$ we deduce that $D_{1} u^{2}=D_{2} u^{1}$ as distributions on the interior of $\operatorname{Dom}(u)$, this and the maximal monotonicity of $u$ easily imply that $u=\partial \varphi$ for some convex function on $\operatorname{Int}(\operatorname{Dom}(u))$.

In the next lemma we provide a simple representation of $G_{u}$ in cylinders $B \times \mathbb{R}^{2}$ corresponding to regions $B$ where $u$ is continuous.

Lemma 3.5. Let $u: \mathbb{R}^{2} \rightarrow P\left(\mathbb{R}^{2}\right)$ be a monotone map, and assume that $B \subset \mathcal{C}_{u}$, with $B$ Borel set with compact closure in the interior of $\operatorname{Dom}(u)$. Then, if

$$
\omega=\omega^{20} d x^{1} \wedge d x^{2}+\sum_{i, j=1}^{2} \omega^{\overline{i j}} d x^{\bar{i}} \wedge d y^{j}+\omega^{02} d y^{1} \wedge d y^{2}
$$

is a bounded Borel 2-form with support contained in $B \times \mathbb{R}^{2}$, we have

$$
\begin{aligned}
G_{u}(\omega)= & \int_{B} \omega^{20}(x, u(x)) d x+\sum_{i, j=1}^{2}(-1)^{i} \int_{B} \omega^{i j}(x, u(x)) d D_{i} u^{j}(x) \\
& +\int_{B} \omega^{02}(x, u(x)) d J u(x) .
\end{aligned}
$$

In addition, if $J u\left\llcorner B \ll \mathscr{L}^{2}\right.$ and $C \subset \Gamma_{u} \cap\left(B \times \mathbb{R}^{2}\right)$ is a Borel set satisfying $\mathscr{L}^{2}\left(\pi_{1}(C)\right)=0$, then

$$
\mathscr{H}^{2}(C)=|D u|\left(\pi_{1}(C)\right) .
$$

Proof. Thanks to Theorem 3.2(i) and Theorem 3.3(iv) we can find a sequence of Lipschitz maps $u_{k}$, such that $\Gamma_{u_{k}} \rightarrow \Gamma_{u}$ in the sense of Kuratowski, $\left|D u_{k}\right| *$ $|D u|$ in the interior of $\operatorname{Dom}(u), \operatorname{det} D u_{k} \mathscr{L}^{2} \stackrel{*}{\rightarrow} J u$ and $G_{u_{k}} \stackrel{*}{\rightarrow} G_{u}$ as currents. Let $A$ be an open set contained in the interior of $\operatorname{Dom}(u)$. If $\omega$ is a smooth 2 -form compactly supported in $A \times \mathbb{R}^{2}$ we have

$$
G_{u}(\omega)=\lim _{k \rightarrow \infty} G_{u_{k}}(\omega)
$$

Splitting $G_{u_{k}}$ (viewed as vector-valued measures) and $G_{u}$ in components and positive and negative parts and doing the same for the coefficients of $\omega$, we are led to the analysis of the limit

$$
\lim _{k \rightarrow \infty} \int g_{k} d \sigma_{k}
$$


where: $\sigma_{k}$ is the positive or negative part of some component of $G_{u_{k}}$, which converge thanks to Lemma 8.1 to the correspondent positive and negative part of $G_{u}$; $g_{k}(x)=f\left(x, u_{k}(x)\right), f$ being the positive or negative part of the correspondent component of $\omega$. Because of (12), the $\Gamma$-limits $g_{ \pm}$in (37) and (39) coincide with $f(x, u(x))$ on $\mathcal{C}_{u}$. As a consequence, formula (11) for the action of $G_{u_{k}}$ and Lemma 8.2 give

$$
\begin{aligned}
\limsup _{k \rightarrow \infty} \mid & G_{u_{k}}(\omega)-\int_{A} \omega^{20}(x, u(x)) d x \\
& +\sum_{i, j=1}^{2}(-1)^{i} \int_{A} \omega^{\bar{i} j}(x, u(x)) d D_{i} u^{j}+\int_{A} \omega^{02}(x, u(x)) d J u \mid
\end{aligned}
$$

can be estimated from above with $C\left(\mathscr{L}^{2}\left(A \backslash \mathcal{C}_{u}\right)+|D u|\left(A \backslash \mathcal{C}_{u}\right)+J u\left(A \backslash \mathcal{C}_{u}\right)\right)$, where $C$ depends only on $\|\omega\|_{\infty}$. Since $B \subset \mathcal{C}_{u}$, combining with (21) and letting $A \downarrow B$ (using the outer regularity of $\mathscr{L}^{2}+|D u|+J u$ ) we get

$$
\begin{aligned}
G_{u}\llcorner B(\omega)= & \int_{B} \omega^{20}(x, u(x)) d x+\sum_{i, j=1}^{2}(-1)^{i} \int_{B} \omega^{i j}(x, u(x)) d D_{i} u^{j} \\
& +\int_{B} \omega^{02}(x, u(x)) d J u .
\end{aligned}
$$

The extension to Borel forms is immediate.

In order to prove (20) first notice that (19) implies that $|D u|\left(\pi_{1}(C)\right) \leq \mathscr{H}^{2}(C)$ without any assumption on the Lebesgue measure of $\pi_{1}(C)$. The reverse inequality, follows from the following general fact: if $\mu$ is a vector-valued measure with finite total variation and $T: X \rightarrow Y$ is an injective map, then

$$
\left|T_{\#} \mu\right|=T_{\#}|\mu| \text {. }
$$

Indeed, the inequality $\leq$ is trivial; starting from $\left|S_{\#} v\right| \leq S_{\#}|v|$ with $v=T_{\#} \mu$ and $S: T(X) \rightarrow X$ equal to the inverse of $T$ we get $|\mu| \leq S_{\#}\left|T_{\#} \mu\right|$. Applying $T$ to both sides gives $T_{\#}|\mu| \leq\left|T_{\#} \mu\right|$.

In particular, since, thanks to (19),

$$
G_{u}\left\llcorner B \times \mathbb{R}^{2}=(I d \times u)_{\#}\left(\mathscr{L}^{2}, D_{1} u^{1}, \ldots, D_{2} u^{2}, J u\right)\llcorner B\right.
$$

as vector valued measures and $J u \ll \mathscr{L}^{2}$ we can apply $(22)$ with $T(x)=(x, u(x))$ to obtain that for any Borel set $C \subset \Gamma_{u} \cap\left(B \times \mathbb{R}^{n}\right)$ with negligible projection it holds

$$
\mathscr{H}^{2}(C)=\left|G_{u}\right|(C)=|D u|\left(\pi_{1}(C)\right) .
$$

Proposition 3.6. Let $B \subset \mathbb{R}^{2}$ be a Borel set contained in the interior of $\operatorname{Dom}(u)$. Then $\mathscr{H}^{2}\left(V \cap\left(B \times \mathbb{R}^{2}\right)\right)=0$ implies $\left|D^{s} u\right|(B)=0$. A partial converse holds if $B$ is open, namely $u \in W_{\mathrm{loc}}^{1,1}\left(B ; \mathbb{R}^{2}\right)$ implies $\mathscr{H}^{2}\left(V_{u} \cap\left(B \times \mathbb{R}^{2}\right)\right)=0$. 
PROOF. Let us assume by contradiction that $V \cap\left(B \times \mathbb{R}^{2}\right)$ is $\mathscr{H}^{2}$-negligible, but $\left|D^{s} u\right|(B)>0$. By $(17)$ we get $\mathscr{H}^{2}(\tilde{B})>0$, where $\tilde{B}:=\Gamma_{u} \cap\left(B \times \mathbb{R}^{2}\right)$. Now, by assumption $\mathscr{H}^{2}$-almost all points in $\tilde{B}$ are not vertical, hence (15) gives $\mathscr{L}^{2}(B)>0$, a contradiction.

To prove the second part when $B=A$ is open we need to implement an equation in the spirit of (19) for Sobolev maps $u$, not necessarily continuous, more precisely

$$
G_{u}\left(\varphi d x^{\bar{i}} \wedge d y^{j}\right)=(-1)^{i} \int_{A} \varphi(x, u(x)) D_{i} u^{j}(x) d x
$$

for all bounded Borel $\varphi$ whose support has a projection compactly supported in $A$. To prove this identity, taking the locality of $G_{u}$ into account, we can assume with no loss of generality that $\operatorname{Dom}(u)=\mathbb{R}^{2}$, so that $u$ is locally bounded. Now we approximate $u$ by convolution and use [1, Proposition 1.7] to obtain that the graphs of the mollified functions $u_{\varepsilon}$ converge in the Kuratowski sense to the graph of $u$. From Theorem 3.2(i) we obtain convergence of the currents, so that (23) can be proved by a limiting procedure when $\varphi$ is smooth. When $\varphi$ is bounded Borel we perform an additional approximation. Now, suppose by contradiction that $\mathscr{H}^{2}\left(V_{u} \cap\left(A \times \mathbb{R}^{2}\right)\right)>0$. Then, for some $i, j \in\{1,2\}$ there exists a 2-form $\omega=\varphi d x^{\bar{i}} \wedge d y^{j}$ with support contained in $A \times \mathbb{R}^{2}$ such that $G_{u}\left\llcorner V_{u}(\omega)>0\right.$, so that (23) gives

$$
0<G_{u}\left\llcorner V_{u}(\omega)=(-1)^{i} \int_{A \cap \pi_{1}\left(V_{u}\right)} \varphi(x, u(x)) D_{i} u^{j}(x) d x .\right.
$$

This contradicts the fact that $\mathscr{L}^{2}\left(\pi_{1}\left(V_{u}\right)\right)=0$, a simple consequence of $(15)$.

EXAMPLE 3.7. If $u \in W_{\text {loc }}^{1,1}$ nothing can be said in general about $\mathscr{H}^{2}\left(V \backslash V_{u}\right)$, to see this consider the subdifferential of $\varphi(x)=|x|$, namely

$$
\partial \varphi(x):=u(x)= \begin{cases}x /|x| & \text { if } x \neq 0 \\ \{p:|p| \leq 1\} & \text { if } x=0\end{cases}
$$

Then $u \in W_{\text {loc }}^{1,1}\left(\mathbb{R}^{2} ; \mathbb{R}^{2}\right)$ but $\mathscr{H}^{2}\left(V \backslash V_{u}\right)>0$.

\section{Singular part of $D u$ and Singular gradients of $f$}

Let $u: \mathbb{R}^{2} \rightarrow \mathbb{R}^{2}$ be a monotone gradient and assume that for some Borel set $B \subset \mathcal{C}_{u}$ the measure $J u\left\llcorner B\right.$ is absolutely continuous with respect to $\mathscr{L}^{2}$. Under these assumption we are going to investigate more closely the structure of the orienting 2-vector $\xi_{u}$ to $\Gamma_{u}$ at points $(x, u(x))$ with $x \in B$. Recall that this concept is well defined, thanks to the fact that $\Gamma_{u}$ in the system of coordinates $\left(e_{1}^{\prime}, e_{2}^{\prime}, \varepsilon_{1}^{\prime}, \varepsilon_{2}^{\prime}\right)$ is the graph of a 1-Lipschitz function $f$. 
Since $u$ is fixed throughout this section we write $\xi=\xi_{u}$ and

$$
\xi=\xi^{20} e_{1} \wedge e_{2}+\sum_{i, j=1}^{2} \xi^{i j} e_{i} \wedge \varepsilon_{j}+\xi^{02} \varepsilon_{1} \wedge \varepsilon_{2} .
$$

We have proved in Proposition 3.6 that, for $B$ contained in the interior of $\operatorname{Dom}(u)$, if $\left|D^{s} u\right|(B)>0$ implies $\mathscr{H}^{2}\left(V \cap\left(B \times \mathbb{R}^{2}\right)\right)>0$. Now we prove that $J u\left\llcorner B \ll \mathscr{L}^{2}\right.$ implies $\mathscr{H}^{2}\left(\left(V \backslash V_{u}\right) \cap\left(B \times \mathbb{R}^{2}\right)\right)=0$, so that $\left|D^{s} u\right|(B)>0$ implies the stronger conclusion $\mathscr{H}^{2}\left(V_{u} \cap\left(B \times \mathbb{R}^{2}\right)\right)>0$.

Proposition 4.1. If $B \subset \mathbb{R}^{2}$ is Borel set, then

$$
J u\left\llcorner B \ll \mathscr{L}^{2} \quad \text { if and only if } \quad \mathscr{H}^{2}\left(\left(V \backslash V_{u}\right) \cap\left(B \times \mathbb{R}^{2}\right)\right)=0 .\right.
$$

Proof. Assume $J u\left\llcorner B \ll \mathscr{L}^{2}\right.$, let $L:=\left(V \backslash V_{u}\right) \cap\left(B \times \mathbb{R}^{2}\right)$ and set $C:=\pi_{1}(L)$; since $\pi_{1}(V)$ is Lebesgue negligible we get $J u(C)=0$, hence (16) gives

$$
0=J u(C)=\mathscr{L}^{2}\left(\pi_{2}\left(\left(C \times \mathbb{R}^{2}\right) \cap \Gamma_{u}\right)\right)=\int_{\left(C \times \mathbb{R}^{2}\right) \cap \Gamma_{u}}\left|\xi^{02}\right| d \mathscr{H}^{2} .
$$

Since $\left(C \times \mathbb{R}^{2}\right) \cap \Gamma_{u}=L$ is contained in $V \backslash V_{u}$, where $\xi^{02}$ does not vanish, it follows that $L$ is $\mathscr{H}^{2}$-negligible.

In order to show the converse, take a $\mathscr{L}^{2}$-negligible compact subset $C$ of $B$, then

$$
\begin{aligned}
J u(C) & =\mathscr{L}^{2}(u(C))=\int_{\Gamma_{u} \cap\left(C \times \mathbb{R}^{2}\right)}\left|\xi^{02}\right| d \mathscr{H}^{2} \\
& =\int_{\left(\Gamma_{u} \cap V\right) \cap\left(C \times \mathbb{R}^{2}\right)}\left|\xi^{02}\right| d \mathscr{H}^{2}+\int_{\left(\Gamma_{u} \backslash V\right) \cap\left(C \times \mathbb{R}^{2}\right)}\left|\xi^{02}\right| d \mathscr{H}^{2} .
\end{aligned}
$$

Now the first term is zero since, by hypothesis, the set of vertical points such that $\left|\xi^{02}\right|>0$ has zero $\mathscr{H}^{2}$-measure, while the second one vanishes since the set $\left(\Gamma_{u} \backslash V\right) \cap\left(C \times \mathbb{R}^{2}\right)$ has a negligible projection on the first coordinate and $\left|\xi^{20}\right|>0$ on it, so it must be $\mathscr{H}^{2}$-negligible according to (15).

We thus have discovered that, under the additional assumption $J u\left\llcorner B \ll \mathscr{L}^{2}\right.$ and $B$ is contained in the interior of $\operatorname{Dom}(u)$, then $\left|D^{s} u\right|(B)>0$ implies $\mathscr{H}^{2}\left(V_{u} \cap\left(B \times \mathbb{R}^{2}\right)\right)>0$. Now we show that on the projection of $V_{u}$ on the diagonal $\Delta$ (which has positive measure as well, since $\pi_{\Delta}: \Gamma_{f}=\Gamma_{u} \rightarrow \Delta$ is a bi-Lipschitz map) the gradient of $f$ has a particular structure, equivalent to

$$
\left(\begin{array}{cc}
1 & 0 \\
0 & -1
\end{array}\right)
$$

up to a $S O(2)$ rotation. Moreover, we show that this particular structure characterizes the existence of a singular part in the distributional derivative. 
Proposition 4.2. Let $u: \mathbb{R}^{2} \rightarrow P\left(\mathbb{R}^{2}\right)$ be a monotone gradient, $B \subset \mathbb{R}^{2}$ a Borel set satisfying $J u\left\llcorner B \ll \mathscr{L}^{2}\right.$ and let $D \subset \Delta$ be the set of points in $\pi_{\Delta}\left(B \times \mathbb{R}^{2}\right)$ where Df exists and is equivalent to (24) up to a $S O(2)$ rotation. Then, $\left(B \times \mathbb{R}^{2}\right) \cap V_{u}$ coincides up to $\mathscr{H}^{2}$-negligible sets with $\left(D \times \Delta^{\perp}\right) \cap \Gamma_{f}$.

ProOf. First we prove that at totally vertical points the matrix $D f$ has the given structure. We know that at these points we have $\xi=\sum_{i j} \xi^{i j} e_{i} \wedge \varepsilon_{j}$, and we can compare this representation with the one ensured by (9) in the system of coordinates $\left(e_{1}^{\prime}, e_{2}^{\prime}, \varepsilon_{1}^{\prime}, \varepsilon_{2}^{\prime}\right)$ :

$$
\begin{aligned}
\xi=\frac{1}{|\mathcal{M}(D f)|} & \left(e_{1}^{\prime} \wedge e_{2}^{\prime}+D_{e_{2}^{\prime}} f^{1} e_{1}^{\prime} \wedge \varepsilon_{1}^{\prime}+D_{e_{2}^{\prime}} f^{2} e_{1}^{\prime} \wedge \varepsilon_{2}^{\prime}\right. \\
& \left.-D_{e_{1}^{\prime}} f^{1} e_{2}^{\prime} \wedge \varepsilon_{1}^{\prime}-D_{e_{1}^{\prime}} f^{2} e_{2}^{\prime} \wedge \varepsilon_{2}^{\prime}+\operatorname{det} D f \varepsilon_{1}^{\prime} \wedge \varepsilon_{2}^{\prime}\right) .
\end{aligned}
$$

Recalling that $e_{i}^{\prime}$ and $\varepsilon_{i}^{\prime}$ are related to $e_{i}$ and $\varepsilon_{i}$ by (5), we get

$$
\left\{\begin{array}{l}
e_{1}^{\prime} \wedge e_{2}^{\prime}=\frac{1}{2}\left(e_{1} \wedge e_{2}+e_{1} \wedge \varepsilon_{2}-e_{2} \wedge \varepsilon_{1}+\varepsilon_{1} \wedge \varepsilon_{2}\right) \\
e_{1}^{\prime} \wedge \varepsilon_{1}^{\prime}=e_{1} \wedge \varepsilon_{1} \\
e_{1}^{\prime} \wedge \varepsilon_{2}=\frac{1}{2}\left(-e_{1} \wedge e_{2}+e_{1} \wedge \varepsilon_{2}+e_{2} \wedge \varepsilon_{1}+\varepsilon_{1} \wedge \varepsilon_{2}\right) \\
e_{2}^{\prime} \wedge \varepsilon_{1}^{\prime}=\frac{1}{2}\left(e_{1} \wedge e_{2}+e_{1} \wedge \varepsilon_{2}+e_{2} \wedge \varepsilon_{1}-\varepsilon_{1} \wedge \varepsilon_{2}\right) \\
e_{2}^{\prime} \wedge \varepsilon_{2}^{\prime}=e_{2} \wedge \varepsilon_{2} \\
\varepsilon_{1}^{\prime} \wedge \varepsilon_{2}^{\prime}=\frac{1}{2}\left(e_{1} \wedge e_{2}-e_{1} \wedge \varepsilon_{2}+e_{2} \wedge \varepsilon_{1}+\varepsilon_{1} \wedge \varepsilon_{2}\right) .
\end{array}\right.
$$

Hence, taking first the $e_{1} \wedge e_{2}$ component in (25) and then the $\varepsilon_{1} \wedge \varepsilon_{2}$ component (both null, since we are at totally vertical points) we get

$$
1-D_{e_{1}^{\prime}} f^{1}-D_{e_{2}^{\prime}} f^{2}+\operatorname{det} D f=0, \quad 1+D_{e_{1}^{\prime}} f^{1}+D_{e_{2}^{\prime}} f^{2}+\operatorname{det} D f=0 .
$$

Thus we have that $\operatorname{det} D f=-1$ and $\operatorname{tr} D f=0$. In addition, since the operator norm of $D f$ does not exceed 1 (by the bound on the Lipschitz constant), the modulus of the eigenvalues of the symmetric matrix $D f$ is less than 1 . This implies that, up to a $S O(2)$ rotation, $D f$ is representable as in (24).

To obtain the opposite inclusion, let us call $\tilde{D}=D \times \Delta^{\perp}$, we are going to show that

$$
G_{u}\left\llcorner\tilde{D}\left(\varphi d x^{1} \wedge d x^{2}\right)=G_{u}\left\llcorner\tilde{D}\left(\varphi d y^{1} \wedge d y^{2}\right)=0\right.\right.
$$

for any $\varphi \in C_{c}^{\infty}\left(\mathbb{R}^{2} \times \mathbb{R}^{2}\right)$. This implies that at $\mathscr{H}^{2}$-almost every point in $\tilde{D} \cap \Gamma_{u}$ the orienting 2 -vector $\xi$ is of the form

$$
\xi=\sum \xi^{i j} e_{i} \wedge \varepsilon_{j}
$$

and hence $\tilde{D} \cap \Gamma_{u} \subset V_{u} \cap\left(B \times \mathbb{R}^{2}\right)$ up to $\mathscr{H}^{2}$-negligible sets.

In order to prove (26) notice we have the following relations between the dual bases in the system of coordinates $\left(e_{1}, e_{2}, \varepsilon_{1}, \varepsilon_{2}\right)$ and $\left(e_{1}^{\prime}, e_{2}^{\prime}, \varepsilon_{1}^{\prime}, \varepsilon_{2}^{\prime}\right)$ : 


$$
\left\{\begin{array}{l}
d x^{\prime 1}=\frac{1}{\sqrt{2}}\left(d x^{1}+d y^{1}\right) \\
d x^{\prime 2}=\frac{1}{\sqrt{2}}\left(d x^{2}+d y^{2}\right) \\
d y^{\prime 1}=\frac{1}{\sqrt{2}}\left(-d x^{1}+d y^{1}\right) \\
d y^{\prime 2}=\frac{1}{\sqrt{2}}\left(-d x^{2}+d y^{2}\right)
\end{array}\right.
$$

and

$$
\left\{\begin{array}{l}
d x^{\prime 1} \wedge d x^{\prime 2}=\frac{1}{2}\left(d x^{1} \wedge d x^{2}+d x^{1} \wedge d y^{2}-d x^{2} \wedge d y^{1}+d y^{1} \wedge d y^{2}\right) \\
d x^{\prime 1} \wedge d y^{\prime 1}=d x^{1} \wedge d y^{1} \\
d x^{\prime 1} \wedge d y^{\prime 2}=\frac{1}{2}\left(-d x^{1} \wedge d x^{2}+d x^{1} \wedge d y^{2}+d x^{2} \wedge d y^{1}+d y^{1} \wedge d y^{2}\right) \\
d x^{\prime 2} \wedge d y^{\prime 1}=\frac{1}{2}\left(d x^{1} \wedge d x^{2}+d x^{1} \wedge d y^{2}+d x^{2} \wedge d y^{1}-d y^{1} \wedge d y^{2}\right) \\
d x^{\prime 2} \wedge d y^{\prime 2}=d x^{2} \wedge d y^{2} \\
d y^{\prime 1} \wedge d y^{\prime 2}=\frac{1}{2}\left(d x^{1} \wedge d x^{2}-d x^{1} \wedge d y^{2}+d x^{2} \wedge d y^{1}+d y^{1} \wedge d y^{2}\right)
\end{array}\right.
$$

On $D$ it holds $\operatorname{tr} D f=0$ and $\operatorname{det} D f=-1$, hence

$$
\begin{aligned}
0 & =\int_{D} \tilde{\varphi}\left(x^{\prime}, f\left(x^{\prime}\right)\right)\left(D_{e_{1}^{\prime}} f^{1}\left(x^{\prime}\right)+D_{e_{2}^{\prime}} f^{2}\left(x^{\prime}\right)\right) d \mathscr{H}^{2}\left(x^{\prime}\right) \\
& =G_{f}\left\llcorner\tilde{D}\left(\tilde{\varphi}\left(x^{\prime}, y^{\prime}\right)\left(-d x^{\prime 2} \wedge d y^{\prime 1}+d x^{\prime 1}+d y^{\prime 2}\right)\right)\right. \\
& =G_{u}\left\llcorner\tilde{D}\left(\varphi(x, y)\left(-d x^{1} \wedge d x^{2}+d y^{1} \wedge d y^{2}\right)\right)\right.
\end{aligned}
$$

and

$$
\begin{aligned}
0 & =\int_{D} \tilde{\varphi}\left(x^{\prime}, f\left(x^{\prime}\right)\right)\left(1+\operatorname{det} D f\left(x^{\prime}\right)\right) d \mathscr{H}^{2}\left(x^{\prime}\right) \\
& =G_{f}\left\llcorner\tilde{D}\left(\tilde{\varphi}\left(x^{\prime}, y^{\prime}\right)\left(d x^{\prime 1} \wedge d x^{\prime 2}+d y^{\prime 1} \wedge d y^{\prime 2}\right)\right)\right. \\
& =G_{u}\left\llcorner\tilde{D}\left(\varphi(x, y)\left(d x^{1} \wedge d x^{2}+d y^{1} \wedge d y^{2}\right)\right)\right.
\end{aligned}
$$

where $\tilde{\varphi}\left(x^{\prime}, y^{\prime}\right)=\varphi\left(\frac{x+y}{\sqrt{2}}, \frac{-x+y}{\sqrt{2}}\right)$. By the previous equations we obtain (26) and hence the desired inclusion.

In the next theorem, when $B$ is also contained in the interior of $\operatorname{Dom}(u)$ we prove a more precise relation between the Lebesgue measure of the set of points where $D f$ has the special structure and $|D u|$.

TheOREM 4.3. Let $u: \mathbb{R}^{2} \rightarrow P\left(\mathbb{R}^{2}\right)$ be a monotone gradient, $B \subset \mathbb{R}^{2}$ a Borel set contained in $\mathcal{C}_{u}$ and in the interior of $\operatorname{Dom}(u)$ and let $D$ be as in Proposition 4.2. Then $J u\left\llcorner B \ll \mathscr{L}^{2}\right.$ implies

$$
\frac{1}{\sqrt{2}}|D u|\left(B \cap \pi_{1}\left(V_{u}\right)\right) \leq \mathscr{H}^{2}(D) \leq|D u|\left(B \cap \pi_{1}\left(V_{u}\right)\right) .
$$

In particular, since $\pi_{1}\left(V_{u}\right)$ is $\mathscr{L}^{2}$-negligible, $\left|D^{s} u\right|(B)>0$ if and only if $\mathscr{H}^{2}(D)>0$. 
ProOF. Since $f$ is a 1-Lipschitz map we have

$$
\mathscr{H}^{2}(D) \leq \mathscr{H}^{2}\left(\left(D \times \Delta^{\perp}\right) \cap \Gamma_{f}\right) \leq \sqrt{2} \mathscr{H}^{2}(D),
$$

and by applying first Proposition 4.2 we obtain

$$
\left.\mathscr{H}^{2}\left(\left(D \times \Delta^{\perp}\right) \cap \Gamma_{f}\right)=\mathscr{H}^{2}\left(\left(B \times \mathbb{R}^{2}\right) \cap V_{u}\right)=|D u|\left(\pi_{1}\left(B \times \mathbb{R}^{2}\right) \cap V_{u}\right)\right),
$$

where the last equality follows from (20). The inequalities (28) follow immediately from (29) and (30).

In the next lemma we relate, in a pointwise way, bounds on the Jacobian of $u$ to bounds on trace and Jacobian of $D f$. In the subsequent proposition we prove the converse in an integral form, so that $J u$ is bounded also as a measure.

Lemma 4.4. Suppose $u: \mathbb{R}^{2} \rightarrow P\left(\mathbb{R}^{2}\right)$ is a monotone gradient and let $x$ be a differentiability point of $u$, with

$$
\frac{1}{L} \leq \operatorname{det} \nabla u(x) \leq L
$$

for some $L>1$. Let us consider the 1-Lipschitz map $f$ which corresponds to $u$ under the Cayley transformation and suppose that $f$ is differentiable at $x^{\prime}:=$ $(x+u(x)) / \sqrt{2}$. If we denote $T=\operatorname{tr} D f\left(x^{\prime}\right)$ and $J=\operatorname{det} D f\left(x^{\prime}\right)$, then

$$
\frac{(L+1)}{(L-1)}|T| \leq 1+J \text {. }
$$

Proof. We have

$$
\frac{u-I d}{\sqrt{2}}=f\left(\frac{u+I d}{\sqrt{2}}\right)
$$

thus

$$
\nabla(u-I)(x)=D f\left(x^{\prime}\right)(\nabla u+I)(x)
$$

(where $I$ is the identity matrix) and so $\nabla u(x)=\left(D f\left(x^{\prime}\right)-I\right)^{-1}\left(D f\left(x^{\prime}\right)+I\right)$. If $\lambda_{1}, \lambda_{2} \in[-1,1]$ are the eigenvalues of $D f\left(x^{\prime}\right)$, taking determinants we must have

$$
\frac{1}{L} \leq \frac{\left(1+\lambda_{1}\right)\left(1+\lambda_{2}\right)}{\left(1-\lambda_{1}\right)\left(1-\lambda_{2}\right)} \leq L .
$$

Notice that $\left(1-\lambda_{1}\right)\left(1-\lambda_{2}\right)>0$ and write the previous inequality as

$$
\frac{1}{L} \leq \frac{1+T+J}{1-T+J} \leq L .
$$


The first inequality gives

$$
(1-T+J) \leq L(1+T+J)
$$

which is equivalent to $T(L+1) \geq(1+J)(1-L)$. Therefore, since $L>1$ we get

$$
-\frac{(L+1)}{(L-1)} T \leq 1+J
$$

The second inequality gives

$$
(1+T+J) \leq L(1-T+J)
$$

that is equivalent to $(L+1) T \leq(L-1)(1+J)$. As before, it follows that

$$
\frac{(L+1)}{(L-1)} T \leq 1+J
$$

and the thesis follows.

Using the elementary computations in the previous lemma we see that the condition $(L+1)|T| \leq(L-1)(1+J)$ can be written in the equivalent and nicer form

$$
\frac{1}{L}\left(1-\lambda_{1}\right)\left(1-\lambda_{2}\right) \leq\left(1+\lambda_{1}\right)\left(1+\lambda_{2}\right) \leq L\left(1-\lambda_{1}\right)\left(1-\lambda_{2}\right)
$$

where $\lambda_{1}, \lambda_{2}$ are the eigenvalues of $D f$.

Proposition 4.5. Suppose that the 1-Lipschitz function $f$ satisfies

$$
\frac{1}{L} \operatorname{det}(\operatorname{Id}-D f) \leq \operatorname{det}(\operatorname{Id}+D f) \leq L \operatorname{det}(\operatorname{Id}-D f)
$$

$\mathscr{L}^{n}$-almost everywhere on a Borel $B \subset \Delta$. Then

$$
\frac{1}{L} \mathscr{L}^{2}\left\llcorner A \leq J u\left\llcorner A \leq L \mathscr{L}^{2}\llcorner A\right.\right.
$$

on the Ju-measurable set $A:=(\operatorname{Id}+u)^{-1}(\sqrt{2} B)=(\operatorname{Id}-f)(B) / \sqrt{2}$.

PROOF. Let $\varphi(x)$ be a Borel nonnegative function whose support is contained in $A$. Let us define $\tilde{\varphi}\left(x^{\prime}, y^{\prime}\right)=\varphi\left(\left(x^{\prime}-y^{\prime}\right) / \sqrt{2}\right)=\varphi(x)$. An easy calculation shows that $\tilde{\varphi}$ is supported in $B \times \mathbb{R}^{2}$, hence 


$$
\begin{aligned}
0 \geq & \frac{1}{2} \int_{B} \tilde{\varphi}\left(x^{\prime}, f\left(x^{\prime}\right)\right)\left(\frac{1}{L} \operatorname{det}\left(\operatorname{Id}-D f\left(x^{\prime}\right)\right)-\operatorname{det}\left(\operatorname{Id}+D f\left(x^{\prime}\right)\right)\right) d x^{\prime} \\
= & \frac{1}{L}\left\langle G_{f}, \tilde{\varphi}\left(x^{\prime}, y^{\prime}\right) \frac{d x^{\prime 1}-d y^{\prime 2}}{\sqrt{2}} \wedge \frac{d x^{\prime 2}-d y^{\prime 2}}{\sqrt{2}}\right\rangle \\
& -\left\langle G_{f}, \tilde{\varphi}\left(x^{\prime}, y^{\prime}\right) \frac{d x^{\prime 1}+d y^{\prime 2}}{\sqrt{2}} \wedge \frac{d x^{\prime 2}+d y^{\prime 2}}{\sqrt{2}}\right\rangle \\
= & \frac{1}{L}\left\langle G_{u}, \varphi(x) d x^{1} \wedge d x^{2}\right\rangle-\left\langle G_{u}, \varphi(x) d y^{1} \wedge d y^{2}\right\rangle \\
= & \frac{1}{L} \int_{A} \varphi(x) d x-\int_{A} \varphi(x) d J u(x),
\end{aligned}
$$

where in the last equality we used (18). Since $\varphi$ is arbitrary, this proves the first inequality in (32), the second one follows along the same lines.

\section{5. $C^{1}$ Regularity AND strictly COnvexity of Alexandrov solutions OF Monge-Ampére EQUATIONS}

In this section we briefly recall the proof of the $C^{1}$ regularity of solutions of the Monge-Ampére inequalities

$$
\frac{1}{L} \mathscr{L}^{2}\left\llcorner\Omega \leq\left(\operatorname{det} D^{2} \varphi\right)\left\llcorner\Omega \leq L \mathscr{L}^{2}\llcorner\Omega\right.\right.
$$

Here $\varphi: \mathbb{R}^{2} \rightarrow \mathbb{R} \cup\{+\infty\}$ is a convex function, $\Omega$ is an open set of $\mathbb{R}^{2}$ contained in the domain of $\varphi, L \geq 1$ and $\operatorname{det} D^{2} \varphi$ is the Monge-Ampére measure, corresponding to the Jacobian measure of $\partial \varphi$, see Section 3. Since we are only interested in the continuity of the subdifferential map we give here a proof whose strategy can be summarized in the following way: first of all equation (33) implies the strict convexity of $u$ in the sense that if $p \in \partial \varphi(x)$ then

$$
\varphi(y)>\varphi(x)+\langle p, y-x\rangle \text { for any } y \neq x \text {. }
$$

This implies that $\partial \varphi^{*}$ is univalued and hence continuous. Then we show that if $\varphi$ satisfies $(33)$ then $\varphi^{*}$ satisfies a similar property.

Proposition 5.1 ([6]). If $\left(\operatorname{det} D^{2} \varphi\right)\left\llcorner\Omega \geq c \mathscr{L}^{2}\llcorner\Omega\right.$ for some $c>0$ then $\varphi$ is strictly convex in $\Omega$.

ProOF. By the convexity of $\varphi$, if strict convexity fails at some point $x$, the contact set $\{y: \varphi(y)=\varphi(x)+\langle p, y-x\rangle\}$ contains a small segment touching $x$. Up to scaling and subtracting an affine function we can suppose that $x=0$ and that $\varphi \equiv 0$ on $\{0\} \times[-2,2] \subset \Omega$. Let $R=[h, 2 h] \times[-1,1]$, then (denoting with $\operatorname{det} \nabla^{2} \varphi$ the density with respect to $\mathscr{L}^{2}$ of the measure $\operatorname{det} D^{2} \varphi$ ) 


$$
\begin{aligned}
2 \sqrt{c} h & \leq 2 \int_{R} \sqrt{\operatorname{det} \nabla^{2} \varphi} d x d y \leq \int_{R}\left(\frac{1}{t} D_{x x} \varphi+t D_{y y} \varphi\right) d x d y \\
& =\frac{1}{t} \int_{-1}^{1}\left(D_{x} \varphi(h, y)-D_{x} \varphi(2 h, y)\right) d y+t \int_{h}^{2 h}\left(D_{y} \varphi(x, 1)-D_{y} \varphi(x,-1)\right) d x \\
& :=\frac{1}{t} I_{1}(h)+t I_{2}(h),
\end{aligned}
$$

where the last equality must be understood in the sense of traces since the subdifferential of a convex function coincides with an (univalued) $B V$ function $\mathscr{H}^{n-1}$-almost everywhere. Now by the continuity of left and right derivatives of convex functions and the local Lipschitz property of $\varphi$ we have that $I_{1}$ goes to zero as $h \rightarrow 0$, while $I_{2} \leq C h^{2}$ since for any $x \in[h, 2 h]$ and $y \in[-1,1]$

$$
D_{y} \varphi(x, y) \leq \max _{x \in[h, 2 h]}\left[\max _{y \in[-2,2]} \varphi(x, y)-\min _{y \in[-2,2]} \varphi(x, y)\right] \leq C h .
$$

Choosing $t=\sqrt{I_{1}} / h$ we obtain a contradiction as $h \rightarrow 0$.

LemMA 5.2. If $\varphi$ satisfies $\left(\operatorname{det} D^{2} \varphi\right)\left\llcorner\Omega \leq C \mathscr{L}^{2}\left\llcorner\Omega\right.\right.$ for some $C>0$, then $\varphi^{*}$ satisfies

$$
\left(\operatorname{det} D^{2} \varphi^{*}\right)\left\llcorner\varphi(\Omega) \geq \frac{1}{C} \mathscr{L}^{2}\llcorner\partial \varphi(\Omega) .\right.
$$

Proof. Recall that $(\partial \varphi)^{-1}=\partial \varphi^{*}$ as multivalued functions. Then, if $A \subset \partial \varphi(\Omega)$ we have $A \subset \partial \varphi\left(\Omega \cap \partial \varphi^{*}(A)\right)$ and hence

$$
\mathscr{L}^{2}(A) \leq \mathscr{L}^{2}\left(\partial \varphi\left(\Omega \cap \partial \varphi^{*}(A)\right)\right) \leq C \mathscr{L}^{2}\left(\partial \varphi^{*}(A)\right) .
$$

Since it is well-known that strict convexity of $\varphi^{*}$ implies $C^{1}$ regularity of $\varphi$, to conclude we only have to check that $\partial \varphi(\Omega)$ is open, in order to apply Proposition 5.1 to $\varphi^{*}$.

Lemma 5.3. Let $\varphi: \mathbb{R}^{2} \rightarrow \mathbb{R} \cup\{+\infty\}$ be strictly convex in an open set $\Omega$ contained in its domain. Then $\partial \varphi(\Omega)$ is open.

ProOF. It is obviously sufficient to show that if $0 \in \partial \varphi(\Omega)$ then $B_{\varepsilon}(0) \subset \partial \varphi(\Omega)$ for some $\varepsilon>0$. Let $x \in \Omega$ be such that $0 \in \partial \varphi(x)$ and let $r>0$ be such that $\bar{B}_{r}(x) \subset \Omega$. Assuming with no loss of generality that $\varphi(x)=0$, by strict convexity of $\varphi$ we have that $\alpha:=\min _{\partial B_{r}(x)} \varphi>0$. Let now $q \in \mathbb{R}^{2}$ with $|q|<\alpha /(2 r)$ and consider the function $g_{q}(y)=\varphi(y)-\langle q, y\rangle$. If $x_{q} \in \bar{B}_{r}(x)$ is its minimum point, we claim that $x_{q} \notin \partial B_{r}(x)$, this completes the proof since $x_{q} \in B_{r}(x)$ implies $q \in \partial \varphi\left(B_{r}(x)\right)$ and, since $q$ is arbitrary, $B_{\alpha /(2 r)}(0) \subset \partial \varphi(\Omega)$. To prove the claim, just notice that $g_{q}(0)=0$ while on $\partial B_{r}(x)$ we have

$$
g(y) \geq \alpha-|q| r>\frac{\alpha}{2}>0 .
$$




\section{EquiVALENCE OF THE PROBLEMS AND RELATIONS WITH OPTIMAL}

In this section we gather the results of the previous section and we show that the two problems are equivalent.

So, let $u$ be as in Problem 1 with $u \notin W_{\text {loc }}^{1,1}\left(A ; \mathbb{R}^{2}\right)$ and consider the function $f$ defined on the set $B:=(\operatorname{Id}+u) / \sqrt{2}(A)$, notice that this set is open thanks to the strict monotonicity of $(\mathrm{Id}+u)$ and Lemma 5.3. We claim that $D f \in \mathcal{A}$ almost everywhere in $B$. From Lemma 4.4 we know that this is true on $(\operatorname{Id}+u) / \sqrt{2}\left(D_{u}\right)$, where $D_{u}$ is the set of differentiability points of $u$ in $A$. From Theorem 3.3 (ii) we know that

$$
(\operatorname{Id}+u) / \sqrt{2}\left(D_{u}\right) \supset \pi_{\Delta}\left(\left(\Gamma_{u} \backslash V\right) \cap \mathcal{D} \cap\left(A \times \mathbb{R}^{2}\right)\right)
$$

up to $\mathscr{L}^{2}$-negligible sets. Proposition 4.2 implies that $D f \in \mathcal{A}$ also on $\pi_{\Delta}\left(V_{u} \cap\left(A \times \mathbb{R}^{2}\right)\right)$, since trivially $\mathcal{S} \subset \mathcal{A}$. Our claim now follows since the previous inclusions yield that $\{D f \notin \mathcal{A}\}$ is contained up to $\mathscr{L}^{2}$-negligible sets in

$$
\pi_{\Delta}\left(\Gamma_{u} \cap\left(V \backslash V_{u}\right) \cap \mathcal{D} \cap\left(A \times \mathbb{R}^{2}\right)\right) \cup \pi_{\Delta}\left(\Gamma_{u} \backslash \mathcal{D}\right) .
$$

The latter sets are $\mathscr{L}^{2}$-negligible since they are the projection of $\mathscr{H}^{2}$-negligible sets, thanks to Proposition 4.1 and the existence of the tangent space $\mathscr{H}^{2}$-almost everywhere.

On the other hand, from the results of the previous section we know that $u$ is continuous (and single-valued) in $A$, hence we can apply Theorem 4.3 (see in particular (28)) to obtain that the set $B \cap\{D f \in \mathcal{S}\}$ has positive $\mathscr{L}^{2}$-measure. Now, if $f$ were affine we can assume up to a rotation, and passing possibly to a connected component of $B$, that

$$
f\left(x^{\prime}, y^{\prime}\right) \equiv\left(x^{\prime}+a,-y^{\prime}+b\right) \quad \text { in } B
$$

for some $a, b \in \mathbb{R}$. By taking the first component in both sides of (13) we get $x=-a / \sqrt{2}$ in $A$, a contradiction (this means that monotone functions corresponding to these functions $f$ have a "thin" domain, contained in $\{x=-a / 2\})$.

Conversely, let $f: B \rightarrow \mathbb{R}^{2}$ be a Lipschitz map satisfying $D f \in \mathcal{A} \mathscr{L}^{2}$-a.e. with $\mathscr{L}^{2}(\{D f \in \mathcal{S}\})>0$. Assuming with no loss of generality that $B$ is convex, we obtain that $f$ is 1-Lipschitz gradient, hence we can apply [11, Proposition 3.6] to extend $f$ to a 1-Lipschitz gradient defined on the whole of $\mathbb{R}^{2}$ (still denoted by $f$ ) and denote by $u$ the corresponding monotone operator which is a subdifferential thanks to Proposition 3.4. From Proposition 4.5 we obtain the two-sided bounds (32) on $J u$, in the set $A:=(\operatorname{Id}+u)^{-1}(\sqrt{2} B)=(\operatorname{Id}-f)(B) / \sqrt{2}$ which is open thanks to Proposition 7.3. Now we can use the results of the previous section to obtain that $u$ is continuous in $A$. Eventually we use Theorem 4.3 again to obtain that $u$ is not locally Sobolev in $A$. 


\subsection{Connection with optimal transportation}

In the sequel we briefly recall the connection of Problem 1 with the optimal transportation, our main reference is [15]. Given two probability measures $\mu$ and $v$ in $\mathbb{R}^{n}$ with finite second moments:

$$
\int_{\mathbb{R}^{n}}|x|^{2} d \mu(x), \quad \int_{\mathbb{R}^{n}}|y|^{2} d v(y)<\infty,
$$

the optimal transportation problem with quadratic cost consists in looking for a Borel measurable map $T: \mathbb{R}^{n} \rightarrow \mathbb{R}^{n}$ which solves the variational problem

$$
\min _{T_{\#} \mu=v} \int_{\mathbb{R}^{n}}|x-T(x)|^{2} d \mu(x) .
$$

Actually it is clear that the above problem could have no solution because there is no admissible map (think for example to the case in which $\mu=\delta_{p}$ and $v=\left(\delta_{q}+\delta_{r}\right) / 2$ with $\left.q \neq r\right)$ or because the infimum is not attained. However, we have the following remarkable result.

THeOREM 6.1. If $\mu \ll \mathscr{L}^{n}$, i.e. $\mu=\varrho_{0} \mathscr{L}^{n}$, then there exists an unique map $T$ solving (35). Moreover, $T$ coincides $\mu$-almost everywhere with the gradient of a convex function $\varphi$. In addition, if $v=\varrho_{1} \mathscr{L}^{n}$, the potential function $\varphi$ satisfies the following Monge-Ampére type equation in a pointwise sense:

$$
\varrho_{1}(\nabla \varphi(x)) \operatorname{det} \nabla^{2} \varphi(x)=\varrho_{0}(x) \quad \text { for } \mu \text {-a.e. } x \in \mathbb{R}^{n} .
$$

In the previous theorem we denoted with $\nabla^{2} \varphi$ the absolutely continuous part with respect to $\mathscr{L}^{n}$ of the distributional derivative $D^{2} \varphi$. Recall also that, according to Theorem 3.3(iii), $\operatorname{det} \nabla^{2} \varphi$ is always equal to the absolutely continuous part of the Monge-Ampére measure

$$
\operatorname{det} D^{2} \varphi=J \partial \varphi .
$$

A natural question left open by the previous theorem is the regularity of the transport map $T$. Without any assumption on the densities $\varrho_{0}, \varrho_{1}$, it is easy to construct examples in which $T$ is even discontinuous, think for example to the case in which $\operatorname{spt} \mu$ is connected, while spt $v$ is not. Less trivial is the fact that discontinuities can persist even in some cases when spt $v$ is simply connected, see [4]. The main intuition of Caffarelli in [4] is however that the right assumption is the convexity of $\operatorname{spt} v$, to be more precise the following theorem holds:

Theorem 6.2. Suppose that $1 / L \leq \varrho_{0}, \varrho_{1} \leq L$ on their supports, that spt $\varrho_{1}$ is convex and that spt $\varrho_{0}$ is the closure of an open set $\Omega$ with $\mathscr{L}^{n}$-negligible boundary. If $\varphi$ is the unique (locally in $\Omega$, up to additive constants) convex function such that $\nabla \varphi_{\#} \mu=v$ then $\varphi$ satisfies the following Monge-Ampére inequalities

$$
\frac{1}{L^{2}} \mathscr{L}^{n}\left\llcorner\Omega \leq \operatorname{det} D^{2} \varphi \leq L^{2} \mathscr{L}^{n}\llcorner\Omega .\right.
$$


In addition

$$
\partial \varphi(\Omega) \subset \operatorname{spt} \varrho_{1}
$$

In particular we recover a sub and supersolution in the sense of Alexandrov and Caffarelli's regularity theory can be applied to deduce that $\varphi$ is a $C^{1, \alpha}$ function, see also Section 5. Under these (minimal) hypotheses, however, it is a still open and interesting the question to deduce at least $W_{\mathrm{loc}}^{2,1}$ regularity of $\varphi$, and this is one of the motivations for Problem 1.

\section{SOME PROPERTIES OF $f$}

To conclude, we prove some properties of a potential solution to Problem 2 and the statement made in the introduction about the absence of rank-one connections in Problem 2.

Let's start with the rank-one connections. Since the involved matrices are symmetric, suffices to consider rank-one segments of the form $b \otimes b$.

Lemma 7.1. If $A \in \mathcal{S}$ then $A+b \otimes b \notin \mathcal{A}$ unless $b=0$.

Proof. Let $v_{1}, v_{2}$ be the orthonormal eigenvalues of $A$ corresponding respectively to 1 and -1 and write $b=b^{1} v_{1}+b^{2} v_{2}$, so that

$$
b \otimes b=\sum_{i, j=1}^{2} b^{i} b^{j} v_{i} \otimes v_{j} .
$$

By the 1-Lipschitz property of the matrices in $\mathcal{A}$ we have

$$
1 \geq\left|(A+b \otimes b) v_{1}\right|^{2}=\left|\left(1+\left|b^{1}\right|^{2}\right) v_{1}+b^{1} b^{2} v_{2}\right|^{2}=\left(1+\left|b^{1}\right|^{2}\right)^{2}+\left(b^{1} b^{2}\right)^{2}
$$

and hence $b^{1}=0$. The matrix $A+b \otimes b$ is diagonal in the basis $\left\{v_{1}, v_{2}\right\}$ with eigenvalues 1 and $-1+\left|b^{2}\right|^{2}$ and the relation

$$
\begin{aligned}
(L+1)\left|b^{2}\right|^{2} & =(L+1)|\operatorname{trace}(A+b \otimes b)| \leq(L-1)(1+\operatorname{det}(A+b \otimes b)) \\
& =(L-1)\left|b^{2}\right|^{2}
\end{aligned}
$$

implies $b^{2}=0$.

Lemma 7.2. There exists a constant $K=K(L)>1$ such that

$$
\|A-S\|^{2} \leq 2 K(-\operatorname{det}(A-S)) \quad \forall A \in \mathcal{A}, \forall S \in \mathcal{S},
$$

where $\|\cdot\|$ denotes the Hilbert-Schmidt norm. 
PROOF. Up to a rotation we can suppose that

$$
A=\left(\begin{array}{cc}
1-\varepsilon_{1} & 0 \\
0 & -\left(1-\varepsilon_{2}\right)
\end{array}\right), \quad S=\left(\begin{array}{cc}
\cos \theta & -\sin \theta \\
-\sin \theta & -\cos \theta
\end{array}\right),
$$

where the condition $A \in \mathcal{A}$ can be read as

(i) $\varepsilon_{1}, \varepsilon_{2} \geq 0$

(ii) $(L+1)\left|\varepsilon_{1}-\varepsilon_{2}\right| \leq(L-1)\left(\varepsilon_{1}+\varepsilon_{2}-\varepsilon_{1} \varepsilon_{2}\right)$.

Moreover we can assume without loss of generality that $\left(1-\varepsilon_{1}\right) \geq-\left(1-\varepsilon_{2}\right)$, that is $2-\varepsilon_{1}-\varepsilon_{2} \geq 0$. With this assumptions in force we have

$$
A-S=\left(\begin{array}{cc}
\left(1-\varepsilon_{1}\right)-\cos \theta & \sin \theta \\
\sin \theta & -\left(\left(1-\varepsilon_{2}\right)-\cos \theta\right)
\end{array}\right) .
$$

A straightforward calculation gives

$$
\|A-S\|^{2}=2\left((1-\cos \theta)\left(2-\varepsilon_{1}-\varepsilon_{2}\right)+\frac{\varepsilon_{1}^{2}+\varepsilon_{2}^{2}}{2}\right)
$$

and

$$
-\operatorname{det}(A-S)=(1-\cos \theta)\left(2-\varepsilon_{1}-\varepsilon_{2}\right)+\varepsilon_{1} \varepsilon_{2}
$$

Now it is clear that the lemma follows if we are able to find a constant $K>1$ such that $\left(\varepsilon_{1}^{2}+\varepsilon_{2}^{2}\right) \leq 2 K \varepsilon_{1} \varepsilon_{2}$. In order to prove the previous inequality notice that we can suppose $\varepsilon_{1} \geq \varepsilon_{2} \geq 0$ and that we only have to find a bound for $\varepsilon_{1} / \varepsilon_{2}$. Condition (ii) above implies that $\varepsilon_{2}=0$ if and only if $\varepsilon_{1}=0$ (this is just another instance of the absence of rank-one connections between $\mathcal{A}$ and $\mathcal{S}$ ) so we can assume $\varepsilon_{2}>0$ and rewrite the condition as

$$
(t-1) \leq \frac{L-1}{L+1}\left(t+1-\varepsilon_{1}\right) \leq \frac{L-1}{L+1}(t+1)
$$

where $t=\varepsilon_{1} / \varepsilon_{2}$. Now it is straightforward to verify that $t \leq L$.

We point out an interesting consequence of the previous lemma: suppose $f: B \rightarrow \mathbb{R}^{2}$ satisfies the differential inclusion on a ball $B$ and $\mathscr{L}^{2}(\{D f \in \mathcal{S}\})>0$. Then, by well-known rigidity properties of $S O(2)$, if $\mathscr{L}^{2}(\{D f \in \mathcal{A} \backslash \mathcal{S}\})=0$ then $f$ is affine (see [14]). So, gradients in $\mathcal{A}$ are attained in a set of positive measure for non trivial maps; on the other hand, for any $S \in \mathcal{S}$ the map $g:=J(f-S x)$, where $J$ is the linear transformation such that $J\left(v^{1}, v^{2}\right)=\left(v^{1},-v^{2}\right)$, is quasiconformal. In particular, by well known properties of quasi-conformal maps (see [12, Theorem 16.10.1]), the set $\{D g=0\}=\{D f=S\}$ must have null Lebesgue measure if $f$ is not affine in $B$. As a consequence, the map can attain a fixed singular gradient only on a set of measure zero unless it is affine. It is easy to 
show that at the level of the map $u$ this means that for any $v \in S^{n-1}$ we have $\left|D^{s} u\right|\left(B_{v}\right)=0$, where

$$
B_{v}:=\left\{x \in B^{\prime}: \frac{d D u}{d|D u|}(x)=v \otimes v\right\} .
$$

This indicates that a negative answer to Problem 1 should involve the construction of a rather complex example.

Another consequence of the previous lemma is that if $f$ satisfies $D f \in \mathcal{A}$ $\mathscr{L}^{2}$-a.e. in $B$, the set $(\mathrm{Id}-f)(B)$ is open if $A$ is open.

Proposition 7.3. Let $f$ be a non locally affine solution of the partial differential inclusion $D f \in \mathcal{A}$ on an open set $B$. Then the set $A:=(\operatorname{Id}-f)(B)$ is open.

Proof. Without loss of generality we can assume that $B$ is convex and that $f$ is not affine in $B$. Since $f$ solves the differential inclusion its gradient is symmetric, moreover $I-D f$ is nonnegative definite, so there exists a convex function $G: B \rightarrow \mathbb{R}$ such that $D G=\mathrm{Id}-f$. Thanks to Lemma 5.3 we have just to prove that $G$ is strictly convex. Suppose this is not the case then up to an affine transformation we can suppose that $G\left(x^{\prime}\right)=a \cdot x^{\prime}$ on $[0,1] \times\{0\} \subset B$ and $G\left(x^{\prime}\right) \geq a \cdot x^{\prime}$ everywhere in $B$ for some vector $a$. Since $G$ is continuously differentiable this implies that $D G=a$ on $[0,1] \times\{0\}$. This means that on $[0,1] \times\{0\}$ the function $f\left(x^{\prime}\right)$ coincides with $x^{\prime}+a$. Now take the matrix $S$ in (24), thanks to Lemma 7.2 we know that the map $g\left(x^{\prime}\right)=J\left(f\left(x^{\prime}\right)-S \cdot x^{\prime}\right)$ is quasi conformal (here again $\left.J\left(v^{1}, v^{2}\right)=\left(v^{1},-v^{2}\right)\right)$, moreover it is constant on the segment $[0,1] \times\{0\}$. Since the inverse image of a point by a non constant quasi conformal map is discrete (see [12, Theorem 16.12.1]) we have that $f\left(x^{\prime}\right)=S \cdot x^{\prime}+a$ everywhere on $B$, a contradiction to the assumption that $f$ is not affine.

\section{Appendix}

LEMмA 8.1. Let $\mu_{k} \in \mathcal{M}\left(\Omega ; \mathbb{R}^{p}\right)$ be a sequence of vector-valued measures with finite total variation in $\Omega \subset \mathbb{R}^{n}$ open, satisfying

$$
\mu_{k} \stackrel{*}{\rightarrow} \mu \quad \text { and } \quad\left|\mu_{k}\right| \stackrel{*}{\rightarrow}|\mu|
$$

Then the positive and negative parts of all components of $\mu_{k}$ weakly ${ }^{*}$-converge to the corresponding parts of $\mu$.

Proof. It is a direct application of Reshetnyak continuity theorem (see for example [2, Proposition 3.15]).

Lemma 8.2. Let $f_{k}: \Omega \rightarrow[0,+\infty)$ be nonnegative Borel functions and define

$$
f_{-}(x):=\inf \left\{\liminf _{k \rightarrow \infty} f_{k}\left(x_{k}\right), x_{k} \rightarrow x\right\} .
$$


Then

$$
\liminf _{k \rightarrow \infty} \int_{\Omega} f_{k} d \mu_{k} \geq \int_{\Omega} f_{-} d \mu
$$

whenever $\mu_{k}$ are nonnegative and $\mu_{k} \stackrel{*}{\rightarrow} \mu$ in $\Omega$.

Proof. By Cavalieri's formula for the integral and Fatou's lemma, suffices to show that $\liminf _{k} \mu_{k}\left(\left\{f_{k}>t\right\}\right) \geq \mu\left(\left\{f_{-}>t\right\}\right)$ for all $t>0$. To this aim, given a compact set $K \subset\left\{f_{-}>t\right\}$, the definition of $f_{-}$immediately implies that there is an open neighborhood $U$ of $K$ such that $U \subset\left\{f_{k}>t\right\}$ for $k$ sufficiently large. Then

$$
\liminf _{k \rightarrow \infty} \mu_{k}\left(\left\{f_{k}>t\right\}\right) \geq \liminf _{k \rightarrow \infty} \mu_{k}(U) \geq \mu(U) \geq \mu(K)
$$

and the conclusion follows letting $K$ increase to $\left\{f_{-}>t\right\}$.

Analogously, if all $f_{k} \geq 0$ are equibounded and supported in an compact set $K \subset \Omega$, then

$$
\limsup _{k \rightarrow \infty} \int_{\Omega} f_{k} d \mu_{k} \leq \int_{\Omega} f_{+} d \mu
$$

where

$$
f_{+}(x):=\sup \left\{\limsup _{k \rightarrow \infty} f_{k}\left(x_{k}\right), x_{k} \rightarrow x\right\} .
$$

\section{REFERENCES}

[1] G. Alberti - L. Ambrosio, A geometric approach to monotone functions in $\mathbb{R}^{n}$, Math. Z., 230 (1999), 259-316.

[2] L. Ambrosio - N. Fusco - D. Pallara, Functions of bounded variation and free discontinuity problems, Oxford Mathematical Monographs. The Clarendon Press, Oxford University Press, New York, 2000.

[3] J.-D. Benamou - Y. Brenier, Weak solutions for the semigeostrophic equation formulated as a couples Monge-Ampere transport problem, SIAM J. Appl. Math., 58 (1998), 1450-1461.

[4] L. Caffarelli, The regularity of mappings with a convex potential, Journal of the AMS, 5 (1992), 99-104.

[5] L. Caffarelli, A note on the degeneracy of convex solutions to Monge-Ampére equations, Commun. in Partial Differential Equations, 18 (1993), 1213-1217.

[6] L. Caffarelli, Some regularity properties of solutions to Monge-Ampére equations, Comm. Pure Appl. Math., 44 (1991), 965-969.

[7] M. Cullen - M. Feldman, Lagrangian solutions of semigeostrophic equations in physical space, SIAM J. Math. Anal., 37 (2006), 1371-1395.

[8] H. Federer, Geometric measure theory, Springer, 1969. 
[9] C. Gutierrez, The Monge-Ampére equation, Birkhäuser, 2001.

[10] M. Giaquinta - G. Modica - J. Souček, Cartesian Currents in Calculus of Variations I, Springer, 1998.

[11] E. Le Gruyer, Minimal Lipschitz extensions to differentiable functions, Geom. Funct. Anal., 12 (2009), 1101-1118.

[12] T. Iwaniec - G. Martin, Geometric function theory and non-linear analysis, Oxford Mathematical Monographs. The Clarendon Press, Oxford University Press, New York, 2001 .

[13] B. KirchHeim, Rigidity and Geometry of Microstructures, Lecture notes, available at http://www.mis.mpg.de/publications/other-series/ln.html

[14] S. Muller, Variational models for microstructure and phase transitions, Lecture notes, available at http://www.mis.mpg.de/publications/other-series/ln.html

[15] C. Villani, Optimal Transport, Old and new, Springer, 2008.

Received 9 May 2011,

and in revised form 12 May 2011.

Luigi Ambrosio

Scuola Normale Superiore p.za dei Cavalieri 7

I-56126 Pisa, Italy

1.ambrosio@sns.it

Guido De Philippis

Scuola Normale Superiore

p.za dei Cavalieri 7

I-56126 Pisa, Italy guido.dephilippis@sns.it

Bernd Kirchheim

Mathematical Institute

University of Oxford

kirchheim@maths.ox.ac.uk 